\title{
Neuromuscular disease, respiratory failure and cor pulmonale
}

\author{
John White, R.E. Bullock ${ }^{1}$, P. Hudgson ${ }^{2}$ and G.J. Gibson \\ Department of Respiratory Medicine, Freeman Hospital, Newcastle upon Tyne, and ${ }^{1}$ Departments of \\ Anaesthetics and ${ }^{2}$ Neurology, Newcastle General Hospital, Newcastle upon Tyne, UK
}

\begin{abstract}
Summary: Respiratory muscle weakness is an uncommon cause of chronic respiratory failure and a rare cause of cor pulmonale. The problem may not be apparent unless specific physical signs are sought or appropriate investigations performed. We present three patients who presented diagnostic difficulty for prolonged periods until the presence of respiratory muscle weakness was considered. Once the diagnosis was established treatment with nocturnal nasal intermittent positive pressure ventilation produced a dramatic improvement in symptoms and allowed a return to a near normal lifestyle.
\end{abstract}

\section{Introduction}

Muscle weakness is rarely considered when a cause of chronic respiratory failure and/or right heart failure is being sought. We report three patients who presented diagnostic difficulty for considerable periods until this possibility was considered.

\section{Case reports}

\section{Case 1}

A 35 year old woman was referred for investigation of respiratory failure. She had always been of short stature and low weight and as a child was never good at sports. She had attended an open-air school because of recurrent chest infections. She had been breathless on exertion for many years.

Following her second pregnancy at age 32 she had had persistent left basal pneumonia and subsequent recurrent respiratory infections. A presumptive diagnosis of bronchiectasis was made; cystic fibrosis was considered and was excluded by a normal sweat test. Finally she was admitted to hospital following a respiratory arrest at home associated with a chest infection. This necessitated prolonged assisted ventilation, latterly via tracheostomy with subsequent slow recovery and eventual weaning from the ventilator.

Correspondence: John E.S. White, M.R.C.P., Regional Cardiothoracic Centre, Freeman Hospital, Newcastle upon Tyne NE7 7DN, UK.

Accepted: 22 April 1992
She was then transferred to our department for further investigation. Further questioning reveale symptoms of morning headache and 'muzzines present for several months. On examination she was thin and wasted; she was able to lie supine without discomfort and with no abdominal wall paradox but she had an audibly weak cough. There was a generalized reduction in muscle bulk and power with no fasciculation or myotonia. Respiratory function tests (Table I) showed severe respiratory muscle weakness with hypercapnia but only mild daytime hypoxaemia. Overnight oximeter recording of oxygen saturation $\left(\mathrm{SaO}_{2}\right)$ however showed profound desaturation, with values during most of the night below $80 \%$ and a nadir of $50 \%$. Polysomnography demonstrated hypoventilation but no periods of apnoea.

A diagnosis was made of congenital myopathy, with profound respiratory muscle weakness and respiratory failure. This was supported by an elevated serum creatine kinase, a characteristic electromyogram and muscle biopsy which showed a marked myopathic process without specific features. She was treated with nocturnal intermittent positive pressure ventilation, initially via the tracheostomy and subsequently by nasal mask. On this treatment there was a gratifying improvement $c$ in symptoms over a number of days and repeat overnight recording of $\mathrm{SaO}_{2}$ showed values for $\mathscr{\mathscr { S }}$ most of the night greater than $90 \%$ with a nadir of 0 $80 \%$ (Table I). She is now back to leading an active life, looking after her family with little restriction in activity. 
Table I Pulmonary function and blood gas data

\begin{tabular}{|c|c|c|c|c|c|c|}
\hline & \multicolumn{2}{|c|}{ Patient 1} & \multicolumn{2}{|c|}{ Patient 2} & \multicolumn{2}{|c|}{ Patient 3} \\
\hline \multicolumn{7}{|l|}{ Spirometric volumes* } \\
\hline $\mathrm{FEV}_{1}$ (litres) & \multicolumn{2}{|c|}{$1.0(40)$} & \multicolumn{2}{|c|}{$0.55(18)$} & \multicolumn{2}{|c|}{$1.2(32)$} \\
\hline FEV, supine (litres) & \multicolumn{2}{|c|}{$0.65(26)$} & \multirow{2}{*}{\multicolumn{2}{|c|}{$0.6 \quad(17)$}} & \multicolumn{2}{|c|}{0.6 (16) } \\
\hline VC (litres) & \multicolumn{2}{|c|}{$1.0(35)$} & & & \multicolumn{2}{|c|}{$1.55(33)$} \\
\hline VC supine (litres) & \multicolumn{2}{|c|}{$0.65(22)$} & & & \multicolumn{2}{|c|}{$0.75(16)$} \\
\hline \multicolumn{7}{|c|}{ Maximum respiratory pressures* } \\
\hline$P_{\mathrm{E}} \max \left(\mathrm{cm} \mathrm{H}_{2} \mathrm{O}\right)$ & \multirow{3}{*}{\multicolumn{2}{|c|}{$\begin{array}{l}50(58) \\
15(23)\end{array}$}} & & & \multirow{3}{*}{\multicolumn{2}{|c|}{$\begin{array}{c}100(68) \\
20(19) \\
10(9)\end{array}$}} \\
\hline$P_{\mathrm{I}} \max \left(\mathrm{cm} \mathrm{H} \mathrm{H}_{2} \mathrm{O}\right)$ & & & & & & \\
\hline Pdi sniff $\left(\mathrm{cm} \mathrm{H}_{2} \mathrm{O}\right)$ & & & & & & \\
\hline \multicolumn{7}{|l|}{ Daytime blood gases $\dagger$} \\
\hline & Pre & Post & Pre & Post & Pre & Post \\
\hline $\mathrm{pH}$ & 7.33 & 7.37 & 7.34 & 7.36 & 7.36 & 7.37 \\
\hline$P_{\mathrm{a}} \mathrm{O}_{2}(\mathrm{kPa})$ & 9.0 & 10.3 & 6.3 & 12.2 & 4.62 & 8.2 \\
\hline$P_{\mathrm{a}} \mathrm{CO}_{2}(\mathrm{kPa})$ & 9.0 & 6.6 & 10.0 & 6.37 & 7.09 & 4.6 \\
\hline \multicolumn{7}{|l|}{$\begin{array}{l}\text { Nocturnal } \mathrm{SaO}_{2} \\
\text { Before/during NPPV }\end{array}$} \\
\hline Mean & $65 \%$ & $92 \%$ & $77 \% \ddagger$ & $95 \%$ & $66 \%$ & $90 \%$ \\
\hline Time $\mathrm{SaO}_{2}<90 \%$ & $99 \%$ & $10 \%$ & $99 \% \ddagger$ & $0 \%$ & $99 \%$ & $20 \%$ \\
\hline
\end{tabular}

$\mathrm{FEV}_{1}=$ forced expiratory volume in one second; $\mathrm{VC}=$ vital capacity; $\mathrm{NPPV}=$ nasal positive pressure ventilation; $* \%$ predicted values in parenthesis; †breathing room air; łbreathing 2 litres/minute $\mathrm{O}_{2}$.

Case 2

A 27 year old woman presented with malaise, breathlessness and ankle swelling of 2 weeks' duration. On examination there were signs of right ventricular failure. She gave a history of frequent chest infections and recurrent left lower lobe collapse from the age of one year. A chest radiograph at the age of 12 had been said to show right ventricular enlargement. She had always been weak, underweight and unable to participate in sports and she had difficulty walking upstairs. No organic cause had been found and the diagnosis of anorexia nervosa had been suggested.

At presentation the chest radiograph showed cardiomegaly, bilateral pleural effusions and engorgement of the pulmonary veins. The electrocardiogram showed right ventricular hypertrophy with right axis deviation and an echocardiogram showed right ventricular dilatation; the left ventricle did not appear distended but its function was impaired. The fluid retention was treated with diuretics and she was referred for investigation of a presumed cardiomyopathy. These findings together with the patient's generalized weakness suggested the presence of a congenital myopathy which on muscle biopsy was shown to be nemaline disease.

Respiratory function showed a very severe restrictive ventilatory defect and hypercapnic respiratory failure. Overnight recording of $\mathrm{SaO}_{2}$ confirmed profound desaturation despite breathing supplemental oxygen (Table I). She was treated with nasal positive pressure ventilation (NPPV) at night and for a few hours each afternoon with improvement in her symptoms, physical signs and daytime blood gases (Table I) over a period of a week. Since then she has become more active than ever before, leading an essentially normal life, and her weight has increased from 32 to $51 \mathrm{~kg}$.

\section{Case 3}

A 36 year old man was admitted to hospital with a 10 day history of mild breathlessness and ankle swelling. He had previously been well and able to run his own business, and had not previously consulted a doctor. He was a non-smoker. On examination he was obese and cyanosed, with grossly elevated venous pressure, a loud pulmonary component of the second sound, a fourth heart sound, marked oedema and ascites. Investigation showed polycythaemia, right ventricular hypertrophy on electrocardiogram and hypercapnic respiratory failure. Overnight oximetry showed severe hypoxaemia. He was treated with venesection, oxygen and diuretics with improvement in the signs 
and symptoms, and he was transferred to our unit with a presumptive diagnosis of obstructive sleep apnoea syndrome.

Further enquiry revealed a family history in his younger brother of multiple operations for club foot and his mother had bilateral hand weakness and wasting with a 'steppage' gait. Examination of the patient in the supine posture showed paradoxical motion of the anterior abdominal wall on inspiration. Neurological examination demonstrated slight wasting of the thenar eminence with normal power in the upper limbs, the triceps jerk was just present but the other reflexes were absent, power of ankle flexion was poor and eversion was absent. Sensation was altered up to mid-calf and he demonstrated a 'steppage' gait. A diagnosis was made of hereditary motor-sensory neuropathy Type 1 (Charcot-Marie-Tooth disease) with diaphragmatic weakness, and this was confirmed by further investigation. Echocardiography showed a thickened but well-functioning right ventricle. Nerve conduction velocity studies showed a combined demyelinating/axonal neuropathy with evidence of marked conduction block in some of the nerves, a feature regularly seen in primary demyelinating disorders; this was in keeping with the putative diagnosis and the findings were similar to those found in his relatives. Respiratory function tests (Table I) showed a severe restrictive ventilatory defect with a marked postural fall in vital capacity, poor maximal inspiratory pressure measured at the mouth and a very low transdiaphragmatic pressure (Pdi) during a maximal sniff, confirming severe bilateral diaphragmatic weakness. Polysomnography showed severe hypoventilation without apnoea (Table I).

He was treated with nocturnal NPPV with symptomatic improvement and resolution of clinical signs and was able to return to work within 2 months.

\section{Discussion}

Respiratory failure in patients with neuromuscular disease results from hypoventilation consequent on impaired respiratory muscle function and reduced lung compliance. In each of the three patients described here, weakness had been present for at least 20 years but because of its very slow progression it had gone unrecognized. Each had caused considerable diagnostic difficulty and in two the major respiratory problems came to light only with the development of cor pulmonale.

Unlike the situation with scoliosis, the development of 'right heart failure' seems to be an unusual manifestation of respiratory failure due to respiratory muscle weakness. Both congenital myopathy ${ }^{1,2}$ and hereditary motor-sensory neuro- pathy $^{3-5}$ are recognized, albeit rare, causes of respiratory muscle weakness. Diagnostic difficulty in the patients described here probably arose for: several reasons. The congenital myopathies usually present in childhood or adolescence, so the condition is less likely to be diagnosed in adults. In the case of hereditary motor-sensory neuropathy. diaphragmatic weakness is known to occur but it is rarely sufficient to cause symptoms or the develop ment of respiratory failure.

In the absence of a positive family history $\overrightarrow{0}$ consanguinity, or obvious clinical features of neuromuscular dysfunction, slowly progressive్ muscle weakness often causes diagnostic difficulty Suggestive features include recurrent chest infec 3 tions in childhood and inability to participate ing. games. Breathlessness on exertion may not be $a_{\infty}^{\infty}$ prominent feature as many patients are unable to exercise sufficiently to become breathless ${ }_{\infty}$ Occasional patients present with difficulty in wean ${ }_{0}$ ing from a ventilator after a routine surgicab procedure, or with morning headache and daytime somnolence associated with nocturnal hypo-o ventilation. Examination may reveal selective mus $\frac{?}{0}$ cle wasting and weakness although most of these्ठ patients have 'slim limbs'. Other signs off neuromuscular dysfunction, particularly fascicug tion and myotonia, should always be sought.

Investigation can provide clues to the diagno ofis with, for instance, a chest radiograph reported as showing 'poor inspiratory effort' because of elevation of the diaphragm. The diagnosis of respiratoryo muscle weakness is confirmed by the finding of aष restrictive ventilatory defect and a marked impairment of maximal respiratory pressures. A markedô응 postural fall in vital capacity $(>25 \%)$ is related $\underset{\sigma}{\Im}$ more specifically to weakness or paralysis of the diaphragm. Electrophysiological studies and muscle biopsy provide definitive diagnostic information.

Treatment depends on the underlying cause and 3 . prognosis. Nocturnal ventilatory support, with either positive or negative pressure has become established as effective therapy in appropriate cases $\mathrm{O}$ and its beneficial effects are well recognized ${ }^{6,7} \mathrm{As}^{3}$ well as improving nocturnal pulmonary gaso exchange, its use also leads to increased $\mathrm{PaO}_{2}$ and reduced $\mathrm{PaCO}_{2}$ by day when the patient is breathing $O$ spontaneously. Nasal positive pressure ventilation N is a recent modification of this technique utilizing a N well-fitting nasal mask. ${ }^{8}$ It is generally well ${ }_{\sigma}^{\omega}$ tolerated and has become the method of choice for patients such as those described. ${ }^{9}$ There is little $\frac{0}{\Phi}$ impairment in communication as the mask is $\stackrel{\Phi}{\rightarrow}$ generally worn only whilst the subject is sleeping. Providing patients are able to move their hands to ${ }^{\circ}$ their face the non-invasiveness, simplicity and $\overrightarrow{\mathbb{Q}}$ semi-portability of the equipment makes it the $\frac{\widetilde{\Phi}}{\mathbb{D}}$ most acceptable technique in most circumstances. $\frac{\varrho}{0}$ 
In conclusion, chronic respiratory muscle weakness may be difficult to recognize and should be considered in any patient with cardio-

\section{References}

1. Braun, N.M.T., Arora, N.S. \& Rochester, D.F. Respiratory muscle and pulmonary function in polymyositis and other proximal myopathies. Thorax 1983, 38: 616-623.

2. Harrison, B.D.W., Collins, J.V., Brown, K.G.E. \& Clark, T.J.H. Respiratory failure in neuromuscular diseases. Thorax 1971, 26: 579-584.

3. Nathanson, B., Yu, D.-G. \& Chan, C.K. Respiratory muscle weakness in Charcot-Marie-Tooth disease, a field study. Arch Intern Med 1989, 149: 1389-1391.

4. Eichacker, P.Q., Spiro, A., Sherman, M., Lazar, E., Reichel, J. \& Dodick, F. Respiratory muscle function in hereditary motor sensory neuropathy, type 1. Arch Intern Med 1988, 148: 1739-1740.

5. Laroche, C.M., Carroll, N., Moxham, J., Stanley, N., Courtney Evans, R. \& Green, M. Diaphragm weakness in CharcotMarie-Tooth disease. Thorax 1988, 43: 478-479. respiratory failure in whom the cause is not immediately apparent.

6. Robert, D., Gerard, M., Leger, P. et al. Domiciliary ventilation by tracheostomy for chronic respiratory failure. $\operatorname{Rev~Fr~Mal~}$ Respir 1983, 11: 923-936.

7. Sawicka, E.H., Loh, L. \& Branthwaite, M.A. Domiciliary ventilatory support: an analysis of outcome. Thorax 1983, 43: $31-35$.

8. Carroll, N. \& Branthwaite, M.A. Intermittent positive pressure ventilation by nasal mask: technique and applications. Intensive Care Med 1988, 14: 115-117.

9. Branthwaite, M.A. Non-invasive and domiciliary ventilation; positive pressure techniques. Thorax 1991, 46: 208-212. 